\title{
Molecular epidemiology and virulence characteristics of Staphylococcus aureus nasal colonization in medical laboratory staff: comparison between microbiological and non-microbiological laboratories
}

\author{
Xiaoying Xie ${ }^{1 \dagger}$, Xinlu Dai ${ }^{1+}$, Lijia Ni ${ }^{1}$, Baiji Chen ${ }^{1}$, Zhaofan Luo ${ }^{2}$, Yandan Yao ${ }^{1}$, Xiquan Wu' ${ }^{1}$, Hongyu Li, ${ }^{3,4^{*}}$
} and Songyin Huang ${ }^{1,4^{*}}$

\begin{abstract}
Background: Medical laboratory staff are a high-risk population for colonization of Staphylococcus aureus (S. aureus) due to direct and dense contact with the pathogens; however, there is limited information about this colonization. This study sought to determine the prevalence and molecular characteristics of nasal colonization by S. aureus in medical laboratory staff in Guangzhou, southern China, and to compare the differences between microbiological laboratory (MLS) and non-microbiological laboratory (NMLS) staff.

Methods: S. aureus colonization was assessed by nasal swab cultures from 434 subjects, including 130 MLSs and 304 NMLSs from 33 hospitals in Guangzhou. All S. aureus isolates underwent the antimicrobial susceptibility test, virulence gene detection and molecular typing.

Results: The overall prevalence of S. aureus carriage was 20.1\% (87/434), which was higher in MLSs than in NMLSs (26.2\% vs. 17.4\%, $P<0.05$ ), while the prevalence of Methicillin-resistant S. aureus (MRSA) was similar. Living with hospital staff was associated with $S$. aureus carriage. The majority of the isolates harboured various virulence genes, and those in MLSs appeared less resistant to antibiotics and more virulent than their counterparts. A total of 37 different spa types were detected; among these, t338, t437, t189 and t701 were the most frequently encountered types. T338 was the main spa type contributing to nasal colonization Methicillin-sensitive S. aureus (MSSA) (13.0\%), and t437-SCCmec IV was predominant in MRSA isolates (40\%).
\end{abstract}

Conclusions: These findings provide insight into the risk factors, molecular epidemiology and virulence gene profiles of S. aureus nasal carriage among the medical laboratory staff in Guangzhou.

Keywords: Staphylococcus aureus, Nasal carriage, Medical laboratory, Antimicrobial susceptibility, Virulence genes, spa type

\footnotetext{
* Correspondence: lehyu@126.com; hsongyin@126.com

${ }^{\dagger}$ Equal contributors

${ }^{3}$ Department of Clinical Laboratory, Zengcheng District People Hospital of

Guangzhou, Guangzhou 511300, China

${ }^{1}$ Department of Clinical Laboratory, Sun Yat-Sen Memorial Hospital, Sun

Yat-Sen University, Guangzhou 510120, China

Full list of author information is available at the end of the article
} 


\section{Backgrounds}

Staphylococcus aureus (S. aureus) is a life-threatening pathogen and a part of the commensal flora, and its antimicrobial resistance has been a significant therapeutic challenge. Methicillin-resistant Staphylococcus aureus (MRSA) is of great concern because of its high mortality following treatment failure. S. aureus infections are usually preceded by colonization, which is most often found in the anterior nares [1]. Nasal carriage is associated with $S$. aureus (including MRSA) exposure and an increased risk of transmission and infection $[2,3]$.

$S$. aureus sources and transmission patterns have been extensively investigated, especially in hospital environments [4]. Our previous studies showed possible spread from HA-MRSA (hospital-acquired methicillin-resistant Staphylococcus aureus); to CA-MRSA (community-acquired methicillin-resistant Staphylococcus aureus), which may make the strains more resistant to antibiotics [5]. Therefore, nasal colonization of HA-MRSA strains in HCWs (healthcare workers) may also play a role in MRSA spread from hospital settings to the community [5]. Several studies about healthcare workers have focused on the clinical staff, such as doctors, nurses and general practitioners $[4,6]$, while few studies have investigated the medical laboratory staff in the hospital.

Unlike the clinical staff members who have close contact with patients, medical laboratory staff members are exposed to the infectious materials more often, including patients' blood, fluids and tissues. The medical microbiological laboratory is an important department in the medical lab, and its main function is to isolate, culture and identify the pathogens from the patient specimens. Therefore, the microbiological lab staff members have a higher risk of exposure to $S$. aureus because of their direct and dense contact with pathogens [7]. Therefore, it is important to detect nasal colonization of $S$. aureus among the healthy medical laboratory staff, especially the medical microbiological lab staff.

The objective of this study was to assess the prevalence and factors associated with $S$. aureus and MRSA nasal colonization among medical laboratory staff in hospitals in Guangzhou, China. To the best of our knowledge, this is the first study performed in this population in China. Additionally, some $S$. aureus clones are more virulent than others, although any $S$. aureus genotype can become a pathogen under favourable host conditions. Molecular typing is helpful for supporting infection control measures, investigating suspected outbreaks, and preventing nosocomial transmission [8-11]. Therefore, in this study, all isolates were further genotyped by spa typing, and the MRSA isolates were evaluated with SCCmec typing. The presence of virulence genes was determined to establish their possible relationship to the epidemiological data, including the enterotoxin, haemolysin, $p v l$, tsst-1 and exfoliative genes as well as various adhesive protein producing genes. To date, this is the first study to indicate that medical laboratory staff may have occupational exposure to healthcare-associated $S$. aureus in Guangzhou, China. This knowledge will improve our understanding of the characteristics of S. aureus nasal carriage among healthy medical laboratory staff and provide a possible mode of $S$. aureus transmission in our region.

\section{Methods \\ Population and study design}

A cross-sectional prevalence study was conducted in 33 hospitals' medical laboratories in Guangzhou, China between February 22, 2016, and March 17, 2016. All the subjects were the staff working in medical laboratory; the ones with acute and chronic respiratory tract infection were excluded. As the main metropolises with a large population of more than 10,000,000 in southern China, Guangzhou holds a prominent position in the healthcare of local and surrounding areas. As the main tertiary hospitals, the 33 hospitals are distributed in urban and suburban in Guangzhou, making them representative (Fig. 1).

A total of 434 medical laboratory staff, aged 20-56 years, in the 33 hospitals participated in this study; 130 microbiological laboratory staffs (MLS) were randomly recruited from the microbiological labs, and 304 non-microbiological laboratory staffs (NMLS) were randomly recruited from the non-microbiological labs. All volunteers signed informed consent documents approving the use of their samples for research purposes, and the Ethics Committee of Sun Yat-Sen Memorial Hospital approved the study.

Using a standardized questionnaire, the pertinent demographic, medical information and potential factors that are related to $S$. aureus nasal colonization and transmission were collected from each participant. The "Standard working protection" meant the basic lab rule of hygiene, including hand washing, use of gloves, long sleeves and masks. Before sampling, the subjects were independently required to finish the questionnaires. No surveys were eliminated during data collation, and 434 nasal swabs were sampled.

\section{Bacterial strains}

Both anterior nares were swabbed by rotating a sterile dry cotton swab 5 times inside the nostril. The samples were immediately stored in Copan eSwab Liquid Amies preservation medium (eSwab Collection and Preservation System, Copan Italia, Brescia, Italy) and transported at room temperature to the department of bacteriology in $4 \mathrm{~h}$. The swabs were streaked on blood agar plates at $35{ }^{\circ} \mathrm{C}$ for $24 \mathrm{~h}$. Gram-positive, $\beta$-haemolytic, coagulasepositive isolates were confirmed as $S$. aureus using a Vitek ${ }^{\circ} 2$ microbial identification system (bioMérieux, Marcy l'Etoile, France) according to the manufacturer's instructions. 


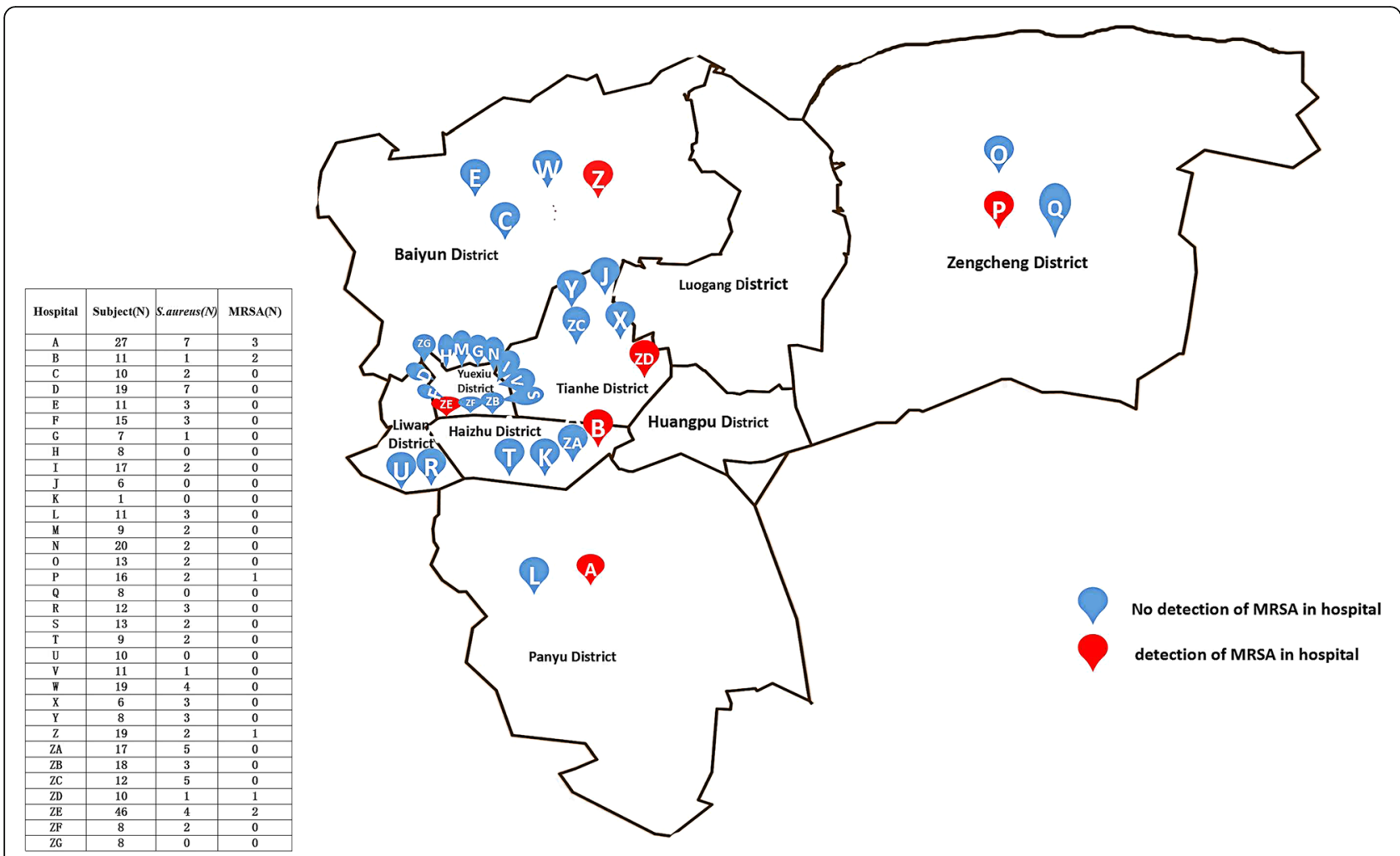

Fig. 1 Map of the 33 hospitals in Guangzhou, China, and the subjects, S. aureus and MRSA isolates distribution

\section{Antimicrobial susceptibility testing}

Antimicrobials used for susceptibility testing included penicillin, erythromycin, clindamycin, tetracycline, cefoxitin, chloramphenicol, rifampicin, ciprofloxacin, gentamicin, trimethoprim/sulfamethoxazole, teicoplanin, linezolid, levofloxacin and vancomycin. Susceptibilities were determined using the disk diffusion method in accordance with the performance standards for antimicrobial susceptibility testing, 26th informational supplement (M100-S26), recommended by the Clinical and Laboratory Standards Institute. The susceptibility for vancomycin was determined using the E-test method. Inducible clindamycin resistance was determined by the D-test. All disks and E-tests were obtained from Oxoid Ltd. (Oxoid, Basingstoke, England), and S. aureus ATCC 25923 and ATCC29213 were used as the quality control strains.

\section{Molecular characterization}

Bacterial DNA was extracted using a DNA extraction kit (Tiangen Biotech, Beijing) with lysostaphin according to the manufacturer's instructions. PCR of the spa locus with subsequent sequencing was performed on the $87 \mathrm{~S}$. aureus isolates, including clustering of the spa types into spa clonal complexes (using the Ridom Staph Type version 1.5 software package, www.spaserver.ridom.de) as previously reported [12]. SCCmec typing of MRSA strains was conducted by a multiplex PCR method as previously described [13]. The presence of the genes that code for Panton-Valentine leukocidin (lukF-PV/lukS-PV or $p v l)$, twelve staphylococcal enterotoxins (sea see, seg sej and sem-seo), two exfoliative toxins (eta and $e t b$ ), four haemolysins ( $\alpha$-hemolysin, $\beta$-hemolysin, $\delta$-hemolysin, and $\gamma$-hemolysin), three biofilm information-related genes (ica $A$, ica $D$ and $b a p$ ), and the toxic shock syndrome toxin (tsst-1) and $m e c \mathrm{~A}$ gene were determined using PCR as previously reported, with some modification $[14,15]$.

\section{Statistical analysis}

In descriptive statistics, the frequency and proportions were calculated for categorical variables. The frequency of the SCCmec type, specimen type and virulence genes were treated as categorical variables. The chi-square or two-sided Fisher's exact test was used to discriminate whether the distributions of the studied genes or types were significantly different between the MLS and NMLS groups. The only continuous variable, the duration of employment, was transformed into a categorical variable using the quartiles of the frequency distribution $(<1,1 \sim$ $5,6 \sim 10,11 \sim 20$, and $>20$ ). The odds ratios (OR), 95\% confidence intervals $(\mathrm{CI})$, and $P$-values were calculated. A finding was considered statistically significant for a two-sided $P$-value $<0.05$. Univariable logistic regression models were applied to determine the independent risk factors. Multiple logistic regression analysis was performed by 
stepwise backward selection of variables with biological plausibility and a significance level $<0.10$ for entry into the model. All statistical analyses were performed using SPSS 19.0 for Windows (IBM).

\section{Results}

\section{S. aureus Nasal colonization}

A total of 434 medical laboratory staff members in the 33 hospitals were enrolled in this study. The demographic characteristics of the study population are shown in Table 1 . The average age of the participating volunteers was $34.2 \pm 12.2$ years, and 187 (43.1\%) of the volunteers were male. $S$. aureus was detected in the nasal swabs of 87 participants $(20.0 \%, 87 / 434)$. The overall prevalence of $S$. aureus nasal colonization was $26.2 \%$ $(34 / 130)$ in MLS, which was higher than that in NMLS $(17.4 \%, 53 / 304)(P=0.03)(\mathrm{OR}=1.677,95 \% \mathrm{CI}: 1.027-$ 2.740). Additionally, there were no differences for other demographic characteristics and potential risk factors between the two comparison groups. A proper nasal cavity cleaning habit could help to decrease the S. aureus nasal carriage [16], but it is interesting to note that the S. aureus nasal carriage rate in people who cleaned their nasal cavity daily or weekly was higher than for those who rarely or never cleaned their nasal cavity $(P=0.032)$ $(\mathrm{OR}=1.753,95 \% \mathrm{CI}: 1.050-2.927)$ in this study, and

Table 1 Prevalence of S. aureus and MRSA carriage by population group and factors associated with nasal carriage

\begin{tabular}{|c|c|c|c|c|c|c|c|c|}
\hline \multirow[t]{2}{*}{ Characteristic (n) } & \multirow{2}{*}{$\begin{array}{l}\text { S. aureus } \\
\mathrm{n}(\%)\end{array}$} & \multicolumn{2}{|c|}{ Univariate logistic } & \multicolumn{2}{|c|}{ Multivariate logistic } & \multirow{2}{*}{$\begin{array}{l}\text { MRSA } \\
\text { n (\%) }\end{array}$} & \multicolumn{2}{|c|}{ Univariate logistic } \\
\hline & & $P$ value & $\mathrm{OR}(95 \% \mathrm{Cl})$ & $P$ value & OR $(95 \% \mathrm{Cl})$ & & $P$ value & $\mathrm{OR}(95 \% \mathrm{Cl})$ \\
\hline \multicolumn{9}{|l|}{ Sex } \\
\hline Male (187) & $42(22.5)$ & 0.155 & $1.414(0.877-2.279)$ & & & $4(2.1)$ & 0.842 & $0.878(0.244-3.157)$ \\
\hline Female (247) & $42(17.0)$ & & & & & $6(2.4)$ & & \\
\hline \multicolumn{9}{|l|}{ Duration of employment } \\
\hline$<1$ year (49) & $9(18.4)$ & 0.149 & $1.790(0.813-3.941)$ & & & $3(6.1)$ & 0.075 & $3.522(0.880-14.093)$ \\
\hline $1-5$ years $(150)$ & $35(23.3)$ & 0.215 & $1.358(0.837-2.202)$ & & & $2(1.3)$ & 0.338 & $0.466(0.098-2.224)$ \\
\hline $6-10$ years $(93)$ & 17(18.2) & 0.631 & $0.866(0.481-1.559)$ & & & $2(2.2)$ & 0.911 & $0.915(0.191-4.383)$ \\
\hline $11-20$ years (85) & $18(21.2)$ & 0.772 & $1.090(0.608-1.953)$ & & & $3(3.5)$ & 0.407 & $1.787(0.452-7.061)$ \\
\hline$>20$ years $(57)$ & $8(14.3)$ & 0.228 & $0.616(0.280-1.354)$ & & & $0(0)$ & - & - \\
\hline \multicolumn{9}{|l|}{ Department } \\
\hline Microbiological laboratory (130) & $34(26.2)$ & 0.039 & $1.677(1.027-2.740)$ & 0.04 & $1.630(1.024-2.597)$ & $2(1.5)$ & 0.492 & $0.578(0.121-2.760)$ \\
\hline Other laboratory (304) & $53(17.4)$ & & & & & $8(2.6)$ & & \\
\hline \multicolumn{9}{|l|}{ Work protection } \\
\hline Standard (354) & $74(20.9)$ & 0.155 & $1.620(0.833-3.151)$ & & & $8(2.3)$ & 0.951 & $0.951(0.197-4.597)$ \\
\hline Non-standard (80) & 13(16.2) & & & & & $2(2.5)$ & & \\
\hline \multicolumn{9}{|l|}{ Nasal cleaning habit } \\
\hline Daily or weekly (106) & $29(27.4)$ & 0.032 & $1.753(1.050-2.927)$ & 0.101 & $1.511(0.923-2.471)$ & $3(2.8)$ & 0.679 & $1.336(0.339-5.259)$ \\
\hline Rarely or never (328) & $58(17.7)$ & & & & & $7(2.1)$ & & \\
\hline \multicolumn{9}{|l|}{ Living with hospital staff } \\
\hline Yes (147) & $40(27.2)$ & 0.008 & $1.909(1.182-3.083)$ & 0.008 & $1.848(1.174-2.901)$ & $3(2.0)$ & 0.794 & $0.833(0.212-3.271)$ \\
\hline No (287) & $47(16.4)$ & & & & & $7(2.4)$ & & \\
\hline \multicolumn{9}{|l|}{ Underlying disease } \\
\hline Yes (65) & $17(26.2)$ & 0.239 & $1.873(0.982-3.573)$ & & & $2(3.3)$ & 0.65 & 1.884(0.386-9.195) \\
\hline No (369) & 70(18.9) & & & & & $8(2.2)$ & & \\
\hline \multicolumn{9}{|l|}{ Hospitalization in past one years } \\
\hline Yes (32) & $9(28.1)$ & 0.157 & $1.085(0.450-2.617)$ & & & $0(0)$ & - & \\
\hline No (402) & $78(19.4)$ & & & & & $10(2.5)$ & & \\
\hline \multicolumn{9}{|l|}{ Current smoking statue } \\
\hline Yes (23) & $5(21.7)$ & 0.837 & $1.114(0.402-3.090)$ & & & $1(4.3)$ & 0.511 & $2.030(0.246-16.748)$ \\
\hline No (411) & $82(20.0)$ & & & & & $9(2.2)$ & & \\
\hline
\end{tabular}

MRSA, methicillin resistant $S$. aureus

Underlying disease: hypertension, diabetes, chronic rhinitis, urticaria, hyperthyroidism; OR odds ratio, $\mathrm{Cl}$ confidence interval 
those living with hospital staff were more likely to be colonized by $S$. aureus in the nasal cavity $(P=0.008)(\mathrm{OR}=$ 1.909, 95\% CI: 1.182-3.083). In multiple logistic regression analysis, nasal colonization of $S$. aureus was also significantly associated with working in a microbiological laboratory and living with hospital staff.

Ten MRSA strains were isolated in this study, two in the MLS group $(1.5 \%, 2 / 130)$ and eight in its counterparts $(2.6 \%, 8 / 304)$, while no significant differences were found between the two groups $(P=0.487)$. MRSA accounted for $11.5 \%$ of all $S$. aureus isolates, and its distribution was centred in three hospitals, Hospital A (three isolates, typed as t008, t437 and t701 respectively), Hospital B (two isolates, typed as $\mathrm{t} 571$ and $\mathrm{t} 441$ respectively) and Hospital ZE (two isolates, typed as $\mathrm{t} 437$ and $\mathrm{t} 037$ respectively), with relative hospital clustering. The distributions of $S$. aureus and MRSA carriers stratified by population characteristics are shown in Table 1 and Fig. 1.

\section{Spa typing and SCCmec typing}

The 87 S. aureus strains were spa typed, resulting in 37 different types, including two new types (Fig. 2 and Additional file 1: Table S1). The largest observed spa clusters were types $\mathrm{t} 338(n=10,11.5 \%)$ and $\mathrm{t} 437(n=10$, $11.5 \%)$, which were followed by $\mathrm{t} 189(n=8,9.2 \%)$ and t701 $(n=8,9.2 \%)$. All types t338 and t189 were MSSA isolates, and type 701 was also mainly found in the MSSA group. MRSA isolates clustered in spa type t437 (4/10, $40 \%)$. All other S. aureus isolates had a distinct spa type. Additionally, two new spa types, t16614 (26-23-12-3412-23-02-02-12-23) (Kreiswirth IDs: TJGBGJAAGJ) and t16615 (11-10-21-17-17-34-24-34-22-25-25) (Kreiswirth IDs: YC2FMMBQBLOO), were found in the MSSA isolates, and they were obtained from nasal swabs of a 26year-old female non-microbiological lab staff member and a 33-year-old male microbiological lab staff member, respectively. There was no clustering of the isolate spa types within different departments.

Based on homology analysis, 37 spa types were grouped into four groups (Fig. 2). Group A contained 12 spa types, including t437, t571 and t002, which evolved based on t002. Group B consisted of ten types, including t701 and t127. Group C contained 13 types, including t189 and t338. The new spa type t16614 formed group D alone. By sequence alignment, the homology of the spa types in the same group was more than $90 \%$. Another new type, t16615, has high homology with $t 701$. The isolates in groups A, B and $\mathrm{C}$ were obtained from both the NMLS and MLS groups, while the isolates from the microbiological laboratory were mainly from groups $\mathrm{A}$ and $\mathrm{C}$, accounting for 85.3\% (29/34). Ten MRSA isolates were dispersed in different groups.

The distribution of SCCmec types in the ten MRSA strains is shown in Table 2. Among these, SCCmec type

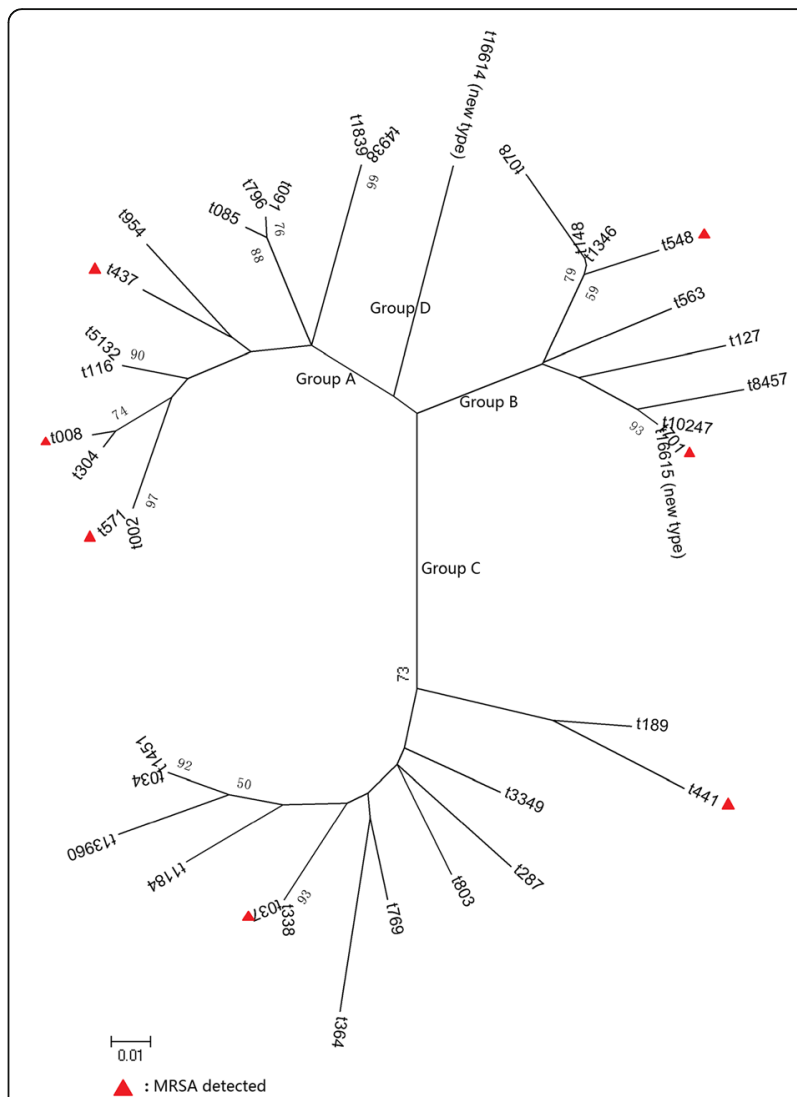

Fig. 2 Thirty-seven spa types grouped into four groups based on DNA microarray analysis. Group A: t002, t571,t304, t008, t116, t5132, t437, t954, t085, t796, t091, t1839, t4938; Group B: t078, t148, t1346, t548, t563, t127, t8457, t10247, t701, t16615(new type); Group C: t189, t441, t3349, t287, t803, t769; t364, t338, t037, t1184, t13960, t034, t1451; Group D: t16614 (new type)

IV was found in eight isolates (80.0\%), MRSA-t437-IV was the most prevalent of all MRSA isolates $(40.0 \%, 4 / 10)$ (Fig. 2 and Table 2). Two MRSA isolates in the NMLS group were SCCmec I MRSA and SCCmec III MRSA, respectively, which were related to hospital-acquired MRSA (HA-MRSA). As a common virulence factor of communityacquired MRSA (CA-MRSA), the $p v l$ gene was positive in $50 \%$ of isolates in the ten MRSA isolates.

\section{Antimicrobial susceptibility}

All isolates were $\beta$-lactamases positive. A high resistance rate to penicillin was detected $(92.0 \%, 80 / 87)$ in the $87 \mathrm{~S}$. aureus isolates, which was followed by the erythromycin $(69.0 \%, 60 / 87)$ and clindamycin $(69.0 \%, 60 / 87)$. All isolates were susceptible to levofloxacin, vancomycin, teicoplanin and linezolid. A total of $44(50.6 \%, 44 / 87)$ isolates were MDRSA [17]; 22 in MLS (64.7\%, 22/34) and 22 in NMLS (41.5\%, 22/53). Penicillin-erythromycin-clindamycin (49.4\%, 43/87) was the predominant resistance profile. Only ten isolates $(11.5 \%, 10 / 87)$ were resistant to cefoxitin, and they 
Table 2 Distribution of the ten MRSA strains in details

\begin{tabular}{|c|c|c|c|c|c|c|c|}
\hline Strain No. & Hospital & Department & Age/Gender & spa type & sccmec & Resistance pattern & Virulence pattern \\
\hline 1 & A & NMLS & 22/Female & t008 & $\mathrm{IVa}$ & DA & sea/tsst-1/hemolysis a/y/d/icaD/pvl \\
\hline 2 & A & NMLS & 20/Female & t437 & IVa & - & sea/seb/she/tsst-1/hemolysis a/y/d/icaD/pvl \\
\hline 3 & A & NMLS & 23/Female & t701 & IVd & $\mathrm{DA} / \mathrm{E} / \mathrm{TE}$ & sea/eta/hemolysis a/y/d/icaD/pvl \\
\hline 4 & B & NMLS & 34/Female & $\mathrm{t} 571$ & IVa & DA/E/RD & seh/tsst-1/hemolysis a/y/d/icaD \\
\hline 5 & B & NMLS & 29/Male & $\mathrm{t} 441$ & I & $\mathrm{DA} / \mathrm{E} / \mathrm{TE}$ & seb/tsst-1/hemolysis $\alpha / \beta / \gamma / \delta / i c a D / p v l$ \\
\hline 6 & ZE & NMLS & 33/Female & t437 & IVa & $\mathrm{DA} / \mathrm{E}$ & seb/seh/tsst-1/hemolysis a/ $\beta / \gamma / \delta / i c a D$ \\
\hline 7 & ZE & NMLS & 32/Female & t037 & III & $\mathrm{DA} / \mathrm{E} / \mathrm{RD}$ & sea/seh/tsst-1/hemolysis a/ $\beta / \gamma / \delta / i c a D$ \\
\hline 8 & P & NMLS & 53/Male & t437 & IVa & $\mathrm{DA} / \mathrm{E} / \mathrm{C}$ & seb/seh/tsst-1/hemolysis a/ $\beta / \gamma / \delta / i c a D$ \\
\hline 9 & Z & MLS & 32/Female & $\mathrm{t} 437$ & IVa & $\mathrm{DA} / \mathrm{E} / \mathrm{TE}$ & 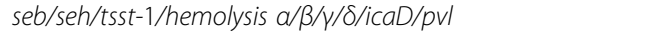 \\
\hline 10 & ZD & MLS & 50/Male & t548 & IVd & $\mathrm{DA} / \mathrm{E} / \mathrm{C} / \mathrm{TE}$ & sed/seg/seh/sei/sej/sen/seo/sem/tsst-1/hemolysis a/y/d/icaD \\
\hline
\end{tabular}

MLS, microbiological lab staff; NMLS, non-microbiological lab staff; -,not detected; DA,clindamycin; TE,tetracycline; E,erythromycin; RD, rifampin; C,chloramphenicol; Sea seo, gene encoding staphylococcal enterotoxins; hla hlg, gene encoding a-haemolysin $\sim$ - hemolysin; tsst-1, gene encoding toxic shock syndrome toxin 1; $p v l$, gene encoding Panton-Valentine leukocidin; icaD, gene relative with the bio-film formation

were confirmed to be MRSA by mecA PCR screening. Nine MRSA isolates were resistant to erythromycin and clindamycin. Only one MRSA isolate was resistant to erythromycin, clindamycin, tetracycline, and gentamycin. Notably, no MRSA was resistant to quinolone in this study. The resistant percentage to 14 antibiotics of the S. aureus isolates is shown in Fig. 3 and Additional file 2: Table S2.

\section{Virulence factors}

Almost all the isolates contained haemolysin gene hla (97.7\%, 85/87), hld (98.9\%, 86/87) and $h l g(98.9 \%, 86 / 87)$ and all isolates in the MLS group were hla-hld-hlg positive. Twelve staphylococcal enterotoxins genes (sea, seg, seh, sem, sen, seo, sec, sei, sej, sem, sen and seo) make up the enterotoxin gene cluster $(e g c)$. Sea, seg, seh, sem, sen,

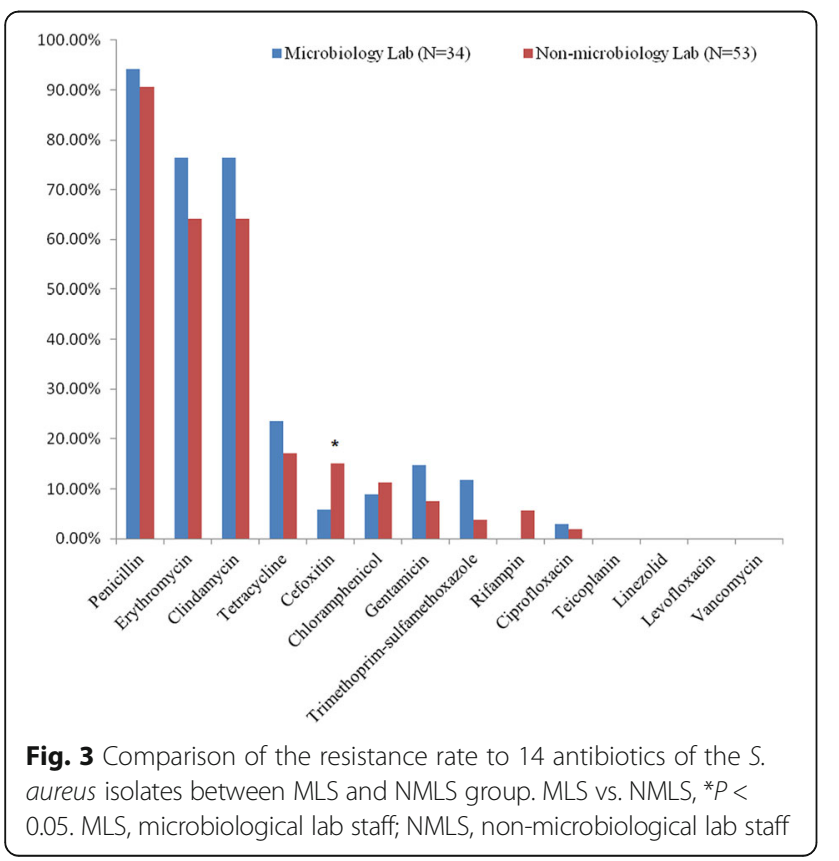

and seo genes were found in more than $20 \%$ isolates in the $87 \mathrm{~S}$. aureus isolates. Additionally, sec, sei and $s e j$ genes were mainly found in the $34 \mathrm{~S}$. aureus isolates in MLS (17.7\%, 23.5\% and $11.8 \%$ respectively), while they were rarely in NMLS. The see gene was not found. Toxic shock syndrome toxin gene (tsst-1) was found in 20 isolates $(23.0 \%)$, and the exfoliative toxins genes (eta or etb) were rarely found (2.3\% and 0 , respectively). The $p v l$ gene was found in 13 isolates (14.9\%), and five isolates were MRSA. The icaA and bap genes were not found, while another biofilm formation related gene icaD widely existed in the S. aureus isolates in this study (Table 3).

Among isolates in MLS, the detection rates of sec and sei were higher than in the NMLS group (17.6\% vs. $1.9 \%$ and $23.5 \%$ vs. $1.9 \%$, respectively) $(P<0.05)$, and all $s e j$ positive isolates were found in the MLS group (11.8\%, 4/ $34)$. On the other hand, the eta gene was only found in isolates of NMLS $(3.8 \%, 2 / 53)$. Furthermore, more than half (51.7\%) of the 87 S. aureus isolates carried more than five virulence genes, and 26.5\% (9/34) of the 34 isolates in MLS group carried more than seven virulence genes, which were obviously higher than that in the NMLS group $(18.9 \%, 10 /$ 53) $(P<0.05)$. Additionally, $80 \%$ of MRSA $(8 / 10)$ carried more than five virulence genes, which was obviously higher than in MSSA $(48.05 \%, 37 / 77)(P<0.05)$ (Table 3$).$

\section{Discussion}

To the best of our knowledge, this is the first study that focused on the prevalence of $S$. aureus and MRSA colonization in medical laboratory staff in China, especially a comparison between microbiological laboratory and nonmicrobiological laboratory staff. This population had a high risk of $S$. aureus colonization. The overall prevalence of $S$. aureus colonization was $20.0 \%$ in this study. Compared with the general population, this is higher than the recorded prevalence among healthy adults in northern China $(16.5 \%)$ and in adults in community settings in southern 
Table 3 Detection of the 23 virulence genes of 87 S. aureus isolates in this study

\begin{tabular}{|c|c|c|c|c|}
\hline \multirow[t]{2}{*}{ Virulence gene } & \multirow{2}{*}{$\begin{array}{l}\text { No. Of positive } \\
\text { isolates } \\
(n=87) \mathrm{n}(\%)\end{array}$} & \multicolumn{2}{|l|}{ No. Distributing in } & \multirow[t]{2}{*}{$P$-value } \\
\hline & & $\begin{array}{l}\text { Microbiology laboratory } \\
(n=34) \mathrm{n}(\%)\end{array}$ & $\begin{array}{l}\text { Other laboratory } \\
(n=53) \mathrm{n}(\%)\end{array}$ & \\
\hline sea & $23(26.4)$ & $8(23.5)$ & $15(28.3)$ & 0.622 \\
\hline seb & $15(17.2)$ & $4(11.7)$ & $11(20.8)$ & 0.279 \\
\hline $\sec$ & $7(8.1)$ & $6(17.6)$ & $1(1.9)$ & 0.008 \\
\hline sed & $5(5.8)$ & $2(5.9)$ & $3(5.7)$ & 0.965 \\
\hline see & $0(0)$ & $0(0)$ & $0(0)$ & - \\
\hline seg & 26(29.9) & $12(35.3)$ & $14(26.4)$ & 0.377 \\
\hline seh & $21(24.2)$ & $9(26.5)$ & $12(22.6)$ & 0.684 \\
\hline sei & $9(10.4)$ & $8(23.5)$ & $1(1.9)$ & 0.001 \\
\hline sej & $4(4.6)$ & $4(11.8)$ & $0(0)$ & 0.011 \\
\hline sem & 29(33.3) & $12(35.3)$ & $17(32.1)$ & 0.756 \\
\hline sen & $24(27.6)$ & $10(29.4)$ & $14(26.4)$ & 0.76 \\
\hline seo & 18(20.7) & $5(14.7)$ & $13(24.5)$ & 0.27 \\
\hline eta & $2(2.3)$ & $0(0)$ & $2(3.8)$ & - \\
\hline etb & $0(0)$ & $0(0)$ & $0(0)$ & - \\
\hline hla & $85(97.7)$ & $34(100)$ & $51(96.2)$ & 0.252 \\
\hline$h l b$ & $38(43.7)$ & $12(35.3)$ & $26(49.1)$ & 0.207 \\
\hline hld & 86(98.9) & $34(100)$ & $52(98.1)$ & - \\
\hline hlg & 86(98.9) & $34(100)$ & $52(98.1)$ & - \\
\hline tsst-1 & $20(23.0)$ & $6(17.7)$ & $14(26.4)$ & 0.343 \\
\hline$p v l$ & $13(14.9)$ & $7(20.6)$ & $6(11.3)$ & 0.237 \\
\hline$i c a A$ & $0(0)$ & $0(0)$ & $0(0)$ & - \\
\hline$i c a D$ & $77(88.5)$ & $31(91.2)$ & $46(86.8)$ & 0.734 \\
\hline bap & $0(0)$ & $0(0)$ & $0(0)$ & - \\
\hline
\end{tabular}

Sea seo, gene encoding staphylococcal enterotoxins; eta and etb, gene encoding exfoliatin; $h l a \sim h l g$, gene encodinga-hemolysin $\sim$-hemolysin; tsst-1, gene encoding toxic shock syndrome toxin 1; pvl, gene encoding Panton-Valentine leukocidin; icaA, ica $D$ and bap, gene relative with the bio-film formation

China (13.7\%) [18, 19], while it agrees with the rates in America and Europe (20-30\%) [20]. Compared with the other HCWs (working mainly in the hospital), the prevalence of $S$. aureus colonization in this study coincides with a review that calculated $23.7 \%$ of $10,589 \mathrm{HCW}$ sorldwide carried $S$. aureus [4]. We expected a higher prevalence of $S$. aureus colonization in the MLS, as there is a higher risk of exposure to $S$. aureus because of the direct, dense contact with pathogens. The MLSs in this study had a high prevalence of $S$. aureus colonization (26.2\%), which was significantly higher than the NMLSs (non-microbiological laboratory staff) $(17.4 \%)$ and higher than that in our other study that focused on the prevalence of $S$. aureus colonization in the nurses and clinical doctors in Guangzhou (21.6\%) [16]. The MRSA colonization rate in this study $(2.3 \%)$ was higher than that in northern Europe $(0.2 \%)$ and southern China $(0.3 \%)$ for healthy adults [21], and it was higher than the $1.0 \%$ in our other study on clinical nurses and doctors [16]. But both the colonization rate of $S$. aureus and MRSA were lower than the research about the carriage among health care professionals (HCPs) attending an international symposium (32.4\% and $5.3 \%$, respectively), $66.88 \%$ of which subjects worked in MLS, and major of which came from Western Europe [7].

Youth, male gender and chronic disease were the most significant risk factors for nasal $S$. aureus colonization in the healthy population according to past studies $[2,18]$; however, in our study, there were no significant differences between different ages and underlying disease conditions. For the medical lab staff, our study found that higher $S$. aureus colonization rates were associated with living with hospital staff. Hospital staff had high risks of pathogen carriage and could act as a "vector" and transmit $S$. aureus to their family, roommates and colleagues [4]. In this study, the prevalence of $S$. aureus colonization appeared to have relative area and hospital clustering, especially MRSA, 70\% of which come from three hospitals, and two isolates were detected in two student technicians in training at Hospital A who were roommates. 
The spa types revealed a broad range of types with a few more prevalent ones, such as t338, t437, t189 and t701, in this study. There were some unique spa clusters in MSSA (t338, t189 and t701) and MRSA (t437) isolates. As the main spa clusters of MSSA in this study, t189 and t338 had high homology, which coincides with the past reports about the molecular epidemiology in seven main cities in China during this time [22]. As a variant of t002, t437 was the predominant spa type of MRSA. According to past reports, MLST CC59-MRSA, which is primarily linked with the spa type 4 437, was the predominant community-associated MRSA clone in Asia, and it represented a genetically tight cluster in Europe [23]. The main SCCmec type of MRSA in this study was SCCmec IV, including IVa and IVd, which showed the isolates were mainly CA-MRSA. Combined with spa typing, MRSA-t437-IV was the most prevalent of all MRSA isolates, which was reported to be a prevalent clone in the community setting in northern China and Taiwan [24, 25]. Two new spa types, t16614 and t16615, were detected in this study; both isolates were detected in MSSA isolates and had characteristics of low virulence and low resistance to antibiotics. Although the spa types are evolving over time in the same person [1, 26, 27], the clustering of the spa type in spa complexes could also be used to describe the transmission direction in our study because all sampling was performed within a short time period (three weeks). There was no obvious difference in the molecular type between MLS and NMLS.

According to the antimicrobial susceptibility test, we found there was a high rate of resistance to penicillin, erythromycin and clindamycin and more than half of the isolates had MDR (multi drug resistance). This resistance pattern is associated with the excessive use of penicillin and macrolides in China and requires substantial attention $[28,29]$. Spa types t189 and t701 appeared relatively sensitive to antibiotics, and their main resistance pattern was to penicillin. While t338 and t437 isolates had higher resistance, and their main resistance pattern was to penicillinclindamycin-erythromycin.

In contrast to the low prevalence of the exfoliative toxins genes (eta or etb) and $p v l$ gene, the enterotoxin genes, tsst1 gene and haemolysin genes were widely distributed among the isolates; more than a half of the isolates carried over five virulence genes. Previous studies have confirmed that the sea and seb genes are the most abundant toxin genes in clinical $S$. aureus isolates from patients in China [5, 30, 31]; in this study, seg and sem $n$ were the predominant enterotoxin genes, which may be related to the different studied populations. Haemolysin of $S$. aureus contributes to bacterio-lyticenzyme release that can damage the nearby organizations through affecting the red blood cells, platelets and neutrophils [32]. Consistent with a report in China [32], almost all isolates contained hla, hld and $h l g$ genes. The tsst- 1 gene was found in $23.0 \%$ of isolates in this study, suggesting that once infection or cross-infection occurred, these patients may have a greater potential for developing fatal toxic shock syndrome (TSS) [33]. Of note, the isolates in MLS appeared to carry more virulence determinants than the ones in NMLS, which may relate to their more heavy and direct exposure to pathogens. MRSA isolates harboured more virulence genes than MSSA isolates in this study. Spa type t189 and t701 isolates harboured fewer virulence genes in this study, and the main toxin pattern was hemolysis $\alpha / \gamma / \delta / i c a D$ (data not shown). As the predominant spa type, t437 appeared more virulent, and it mainly harboured seb/seh/tsst-1/pvl/hemolysis $\alpha / \beta / \gamma / \delta /$ icaD genes. Among the main spa types in this study, the most virulent type was $\mathrm{t} 338$, which harboured more toxin genes (sea/seg/ sem $\sim /$ tsst-1/hemolysis $\alpha / \gamma / \delta /$ icaD). Notably, one MRSA isolate typed as $\mathrm{t} 548$-SCCmec IVd harboured up to 13 virulence genes and appeared to have high resistance to antibiotics; it was isolated from a 50-year-old male microbiology lab staff member who came from hospital ZD, had worked in this department for 27 years and smoked, poor work protection (sometimes didn't wear mask), rarely nasal cleaning and no underlying diseases; additionally, there were HCWs in his family. This colonization of a healthy medical staff member by a high toxin producing and highly resistant strain is worrisome. In the absence of effective function control practices, these strains may become widely disseminated in the hospital and community.

The current study had several limitations. Most importantly, the small size limited the broad representative significance of the research. Second, sampling of only the nostrils without including other body parts may underestimate the frequency of MRSA colonization. Third, MLST typing was not performed in the current study, and it should be conducted in future analyses. Despite the above limitations, our results confirmed the nasal $S$. aureus colonization in a special population, medical lab staff in local region.

\section{Conclusions}

These findings demonstrate an average prevalence of $S$. aureus nasal colonization in medical lab staff as for other HCWs according to the previous reports. Living with hospital staff members and improper nasal hygiene could increase the colonization risk. Some unique spa clusters (t338, t437, t189 and t701), high antibiotic resistance and high virulence were found in the $S$. aureus isolates. Furthermore, MLS had a higher prevalence of $S$. aureus colonization, and the isolates appeared more virulent than the ones in NMLS, which may relate to their more heavy, direct exposure to the pathogens. Some of the many infection prevention partitions, which influence the $S$. aureus/MRSA epidemiology, should be handled by the medical lab staff, especially in the microbiology department, because they have appropriate work protection, nasal clean and hand washing habits. 


\section{Additional files}

Additional file 1: Table S1. Spa types and genetic characteristics of select $\mathrm{S}$. aureus isolates recovered from medical laboratory staff. (DOCX $26 \mathrm{~kb}$ )

Additional file 2: Table S2. The resistance rate to 14 antibiotics of $87 \mathrm{~S}$. aureus isolates. (DOCX $20 \mathrm{~kb})$

\begin{abstract}
Abbreviations
CA-MRSA: community-acquired methicillin-resistant Staphylococcus aureus; CLSI: Clinical and Laboratory Standards Institute; ET: exfoliative toxin; HA-MRSA: hospital-acquired methicillin-resistant Staphylococcus aureus; hla hlg: a-hemolysin y-hemolysin gene; MDRSA: multi-drug resistance S. aureus; MLS: microbiological laboratory; MRSA: Methicillinresistant S. aureus; MSSA: Methicillin-sensitive S. aureus; NMLS: nonmicrobiological laboratory staff; PVL: Panton-Valentine leukocidin gene: S. aureus: Staphylococcus aureus; SCCmec: staphylococcal cassette chromosome mec; SE: staphylococcal enterotoxins; sea seo: staphylococcal enterotoxins A O gene; ST: sequence type; tsst-1: toxic shock syndrome toxin 1 gene
\end{abstract}

\section{Acknowledgments}

We express our thanks to the 33 hospitals in Guangzhou for their participation in this study.

\section{Funding}

This work was supported by grants from the National Natural Science Foundation of China (81272897), the Science and Technology Foundation of the Guangdong Province (2014A050503029), the Sun Yat-sen Initiative Program for Scientific Research (YXQH201701), and the Foundation of Guangzhou Science and Technology Bureau (201400000004-1). This work was supported by Grant [2013]163 from Key Laboratory of Malignant Tumor Molecular Mechanism and Translational Medicine of Guangzhou Bureau of Science and Information Technology. The funders played no role in the study design, data collection and analysis, decision to publish, or preparation of the manuscript.

\section{Availability of data and materials}

All data generated during this study are included in this published article and its additional files. Other relative data analyzed during this study are available from the corresponding author on reasonable request.

\section{Authors' contributions}

SY Huang and $X Y$ Xie contributed to the design of the study and the drafting of the manuscript. XL Dai, LJ Ni, BJ Chen, ZF Luo and XQ Wu performed the experiments, acquired and analyzed the data. YD Yao and $\mathrm{HY}$ Li contributed to revising the manuscript critically for important intellectual content. All authors read and approved the final version of the manuscript.

\section{Ethics approval and consent to participate}

The Ethics Committee of Sun Yat-Sen Memorial Hospital approved the study. Ethics approval number: [2017]伦备第(08)号. All volunteers signed informed consent documents approving the use of their samples for research purposes.

\section{Consent for publication}

Not applicable.

\section{Competing interests}

The authors declare that they have no competing interests.

\section{Publisher's Note}

Springer Nature remains neutral with regard to jurisdictional claims in published maps and institutional affiliations.

\section{Author details}

'Department of Clinical Laboratory, Sun Yat-Sen Memorial Hospital, Sun Yat-Sen University, Guangzhou 510120, China. ${ }^{2}$ Department of Clinical Laboratory, The Seventh Affiliated Hospital of Sun Yat-Sen University, Shenzhen 518071, China. ${ }^{3}$ Department of Clinical Laboratory, Zengcheng District People Hospital of Guangzhou, Guangzhou 511300, China. ${ }^{4}$ Department of Laboratory, Guangdong Provincial Key Laboratory of Malignant Tumor Epigenetics and Gene Regulation, Sun Yat-Sen Memorial Hospital, Sun Yat-Sen University, Guangzhou 510120, China.

Received: 1 May 2017 Accepted: 28 February 2018

Published online: 12 March 2018

\section{References}

1. Kluytmans J, van Belkum A, Verbrugh H. Nasal carriage of Staphylococcus aureus: epidemiology, underlying mechanisms, and associated risks. Clin Microbiol Rev. 1997;10(3):505-20.

2. Botelho-Nevers E, Berthelot P, Verhoeven PO, Grattard F, Cazorla C, Farizon F, Pozzetto B, Lucht F. Are the risk factors associated with Staphylococcus aureus nasal carriage in patients the same than in healthy volunteers? Data from a cohort of patients scheduled for orthopedic material implantation. Am J Infect Control. 2014:42(10):1121-3.

3. Abou Shady HM, Bakr AE, Hashad ME, Alzohairy MA. Staphylococcus aureus nasal carriage among outpatients attending primary health care centers: a comparative study of two cities in Saudi Arabia and Egypt. Braz J Infect Dis. 2015;19(1):68-76.

4. Albrich WC, Harbarth S. Health-care workers: source, vector, or victim of MRSA? Lancet Infect Dis. 2008:8(5):289-301.

5. Xie X, Bao Y, Ouyang N, Dai X, Pan K, Chen B, Deng Y, Wu X, Xu F, Li H, et al. Molecular epidemiology and characteristic of virulence gene of community-acquired and hospital-acquired methicillin-resistant Staphylococcus aureus isolates in sun Yat-sen memorial hospital, Guangzhou, southern China. BMC Infect Dis. 2016:16:339.

6. Hoffmann K, den Heijer CD, George A, Apfalter P, Maier M. Prevalence and resistance patterns of commensal S. Aureus in community-dwelling GP patients and socio-demographic associations. A cross-sectional study in the framework of the APRES-project in Austria. BMC Infect Dis. 2015;15:213.

7. Saadatian-Elahi M, Tristan A, Laurent F, Rasigade JP, Bouchiat C, Ranc AG, Lina G, Dauwalder O, Etienne J, Bes M, et al. Basic rules of hygiene protect health care and lab workers from nasal colonization by Staphylococcus aureus: an international cross-sectional study. PLoS One. 2013;8(12):e82851.

8. Enright MC, Robinson DA, Randle G, Feil EJ, Grundmann H, Spratt BG. The evolutionary history of methicillin-resistant Staphylococcus aureus (MRSA). Proc Natl Acad Sci U S A. 2002;99(11):7687-92.

9. Liu Y, Wang H, Du N, Shen E, Chen H, Niu J, Ye H, Chen M. Molecular evidence for spread of two major methicillin-resistant Staphylococcus aureus clones with a unique geographic distribution in Chinese hospitals. Antimicrob Agents Chemother. 2009;53(2):512-8.

10. Nemeghaire S, Argudin MA, Haesebrouck F, Butaye P. Epidemiology and molecular characterization of methicillin-resistant Staphylococcus aureus nasal carriage isolates from bovines. BMC Vet Res. 2014;10:153.

11. Mirzaii M, Emaneini M, Jabalameli F, Halimi S, Taherikalani M. Molecular investigation of Staphylococcus aureus isolated from the patients, personnel, air and environment of an ICU in a hospital in Tehran. J Infect Public Health. 2015;8(2):202-6

12. den Heijer CD, van Bijnen EM, Paget WJ, Pringle M, Goossens H, Bruggeman CA, Schellevis FG, Stobberingh EE, Team AS. Prevalence and resistance of commensal Staphylococcus aureus, including meticillin-resistant $S$ aureus, in nine European countries: a cross-sectional study. Lancet Infect Dis. 2013;13(5):409-15.

13. Chen $X$, Yang HH, Huangfu YC, Wang WK, Liu Y, Ni YX, Han LZ. Molecular epidemiologic analysis of Staphylococcus aureus isolated from four burn centers. Burns. 2012;38(5):738-42.

14. Sudagidan M, Aydin A. Virulence properties of methicillin-susceptible Staphylococcus aureus food isolates encoding Panton-valentine Leukocidin gene. Int J Food Microbiol. 2010;138(3):287-91.

15. Casagrande Proietti P, Coppola G, Bietta A, Luisa Marenzoni M, Hyatt DR, Coletti M, Passamonti F. Characterization of genes encoding virulence determinants and toxins in Staphylococcus aureus from bovine milk in Central Italy. J Vet Med Sci. 2010;72(11):1443-8. 
16. Chen B, Dai X, He B, Pan K, Li H, Liu X, Bao Y, Lao W, Wu X, Yao Y, et al. Differences in Staphylococcus aureus nasal carriage and molecular characteristics among community residents and healthcare workers at sun Yat-Sen university, Guangzhou, southern China. BMC Infect Dis. 15:303.

17. Ge B, Mukherjee S, Hsu CH, Davis JA, Tran TTT, Yang Q, Abbott JW, Ayers SL, Young SR, Crarey ET, et al. MRSA and multidrug-resistant Staphylococcus aureus in U.S. retail meats, 2010-2011. Food Microbiol. 2017;62:289-97.

18. Yan X, Song Y, Yu X, Tao X, Yan J, Luo F, Zhang H, Zhang J, Li Q, He L, et al. Factors associated with Staphylococcus aureus nasal carriage among healthy people in Northern China. Clin Microbiol Infect. 2015;21(2):157-62.

19. Wang JT, Liao CH, Fang $\mathrm{CT}$, Chie WC, Lai MS, Lauderdale TL, Lee WS, Huang $\mathrm{JH}$, Chang SC. Prevalence of and risk factors for colonization by methicillinresistant Staphylococcus aureus among adults in community settings in Taiwan. J Clin Microbiol. 2009;47(9):2957-63.

20. Mainous AG 3rd, Hueston WJ, Everett CJ, Diaz VA. Nasal carriage of Staphylococcus aureus and methicillin-resistant $S$ aureus in the United States, 2001-2002. Ann Fam Med. 2006;4(2):132-7.

21. Walsh EE, Greene L, Kirshner R. Sustained reduction in methicillin-resistant Staphylococcus aureus wound infections after cardiothoracic surgery. Arch Intern Med. 2011;171(1):68-73.

22. Chen Y, Liu Z, Duo L, Xiong J, Gong Y, Yang J, Wang Z, Wu X, Lu Z, Meng X, et al. Characterization of Staphylococcus aureus from distinct geographic locations in China: an increasing prevalence of spa-t030 and SCCmec type III. PLoS One. 2014;9(4):e96255.

23. Glasner C, Pluister G, Westh H, Arends JP, Empel J, Giles E, Laurent F, Layer F, Marstein L, Matussek A, et al. Staphylococcus aureus spa type t437: identification of the most dominant community-associated clone from Asia across Europe. Clin Microbiol Infect. 2015;21 (2):163.e161-8.

24. Ho CM, Lin CY, Ho MW, Lin HC, Chen CJ, Lin LC, Lu JJ. Methicillin-resistant Staphylococcus aureus isolates with SCCmec type $V$ and spa types $t 437$ or t1081 associated to discordant susceptibility results between oxacillin and cefoxitin, Central Taiwan. Diagn Microbiol Infect Dis. 2016;86(4):405-11.

25. Chen X, Sun K, Dong D, Luo Q, Peng Y, Chen F. Antimicrobial resistance and molecular characteristics of nasal Staphylococcus aureus isolates from newly admitted inpatients. Annals of laboratory medicine. 2016;36(3):250-4.

26. Golubchik T, Batty EM, Miller RR, Farr H, Young BC, Larner-Svensson H, Fung R, Godwin H, Knox K, Votintseva A, et al. Within-host evolution of Staphylococcus aureus during asymptomatic carriage. PLoS One. 2013;8(5):e61319.

27. Halablab MA, Hijazi SM, Fawzi MA, Araj GF. Staphylococcus aureus nasal carriage rate and associated risk factors in individuals in the community. Epidemiol Infect. 2010;138(5):702-6.

28. Mehndiratta PL, Gur R, Saini S, Bhalla P. Staphylococcus aureus phage types and their correlation to antibiotic resistance. Indian J Pathol Microbiol. 2010;53(4):738-41.

29. Treesirichod A, Hantagool S, Prommalikit O. Nasal carriage and antimicrobial susceptibility of Staphylococcus aureus among medical students at the HRH Princess Maha Chakri Sirindhorn medical center, Thailand: a follow-up study J Infect Public Health. 2014;7(3):205-9.

30. He W, Chen H, Zhao C, Zhang F, Li H, Wang Q, Wang X, Wang H. Population structure and characterisation of Staphylococcus aureus from bacteraemia at multiple hospitals in China: association between antimicrobial resistance, toxin genes and genotypes. Int J Antimicrob Agents. 2013;42(3):211-9.

31. Wu D, Li X, Yang Y, Zheng Y, Wang C, Deng L, Liu L, Li C, Shang Y, Zhao C, et al. Superantigen gene profiles and presence of exfoliative toxin genes in community-acquired meticillin-resistant Staphylococcus aureus isolated from Chinese children. J Med Microbiol. 2011;60(Pt 1):35-45.

32. Liu Q, Han L, Li B, Sun J, Ni Y. Virulence characteristic and MLST-agr genetic background of high-level mupirocin-resistant, MRSA isolates from shanghai and Wenzhou, China. PLoS One. 2012;7(5):e37005.

33. Chen X, Wang WK, Han LZ, Liu Y, Zhang H, Tang J, Liu QZ, Huangfu YC, Ni YX. Epidemiological and genetic diversity of Staphylococcus aureus causing bloodstream infection in shanghai, 2009-2011. PLoS One. 2013;8(9):e72811.

\section{Submit your next manuscript to BioMed Central and we will help you at every step:}

- We accept pre-submission inquiries

- Our selector tool helps you to find the most relevant journal

- We provide round the clock customer support

- Convenient online submission

- Thorough peer review

- Inclusion in PubMed and all major indexing services

- Maximum visibility for your research

Submit your manuscript at www.biomedcentral.com/submit
Biomed Central 